\title{
On the Relativistic Separable Functions for the Breakup Reactions
}

\author{
Serge G. Bondarenko ${ }^{1, \star}$, Valery V. Burov ${ }^{1}$, and Elena P. Rogochaya ${ }^{1}$ \\ ${ }^{1}$ Joint Institute for Nuclear Research, 141980 Dubna, Moscow region, Russia
}

\begin{abstract}
In the paper the so-called modified Yamaguchi function for the Bethe-Salpeter equation with a separable kernel is discussed. The type of the functions is defined by the analytic stucture of the hadron current with breakup - the reactions with interacting nucleon-nucleon pair in the final state (electro-, photo-, and nucleon-disintegration of the deuteron).
\end{abstract}

\section{Introduction}

One of the most consistent nucleon-nucleon (NN) interaction theories is based on the solution of the Bethe-Salpeter (BS) equation [1]. In this case, we have to deal with a nontrivial integral equation for the bound state (deuteron) or for the interacting unbound $\mathrm{NN}$ pair.

The approximations based on a kernel with particle exchange are difficult to solve. An effective and solvable approach to the exact solution of the BS equation uses the separable Ansatz for the interaction kernel in the BS equation [2]. In this case one can transform an initial integral equation into a system of linear equations. The parameters of the kernel are obtained by fitting the phase shifts, inelasticity and low-energy parameters for the respective partial-wave states.

The first separable parameterizations were worked out within nonrelativistic models. The separable functions (called form factors) of the interaction kernel used in these models had no poles on the real axis in the relative energy complex plane [3, 4]. However, such poles appeared when the interaction kernel was relativistically generalized.

In some cases they do not prevent to perform the calculations (for example in elastic reactions). However, at high energies, one have to deal with several thresholds corresponding to the production of one, two and more mesons of different types, which is clearly not feasible to deal with. A more practical approach is to employ a phenomenological covariant separable kernel, which do not exhibit the meson-production thresholds and can even be constructed in a singularity-free fashion, using separable form factors and Wick-rotation prescription as it is done in the present paper. Thus, an accurate description of the on-shell nucleon-nucleon data is possible up to quite high energies. One then can hope that the obtained separable interaction kernels have also a reasonable off-shell behavior, so that they can be applied to other reactions as well.

\footnotetext{
${ }^{\star}$ e-mail: bondarenko@jinr.ru
} 


\section{Bethe-Salpeter formalism}

We start with the partial-wave decomposed Bethe-Salpeter equation for the nucleon-nucleon scattering matrix $\mathrm{T}$ (in the rest frame of the two-nucleon system):

$$
\begin{aligned}
t_{L^{\prime} L}\left(p_{0}^{\prime}, p^{\prime}, p_{0}, p ; s\right) & =v_{L^{\prime} L}\left(p_{0}^{\prime}, p^{\prime}, p_{0}, p ; s\right) \\
& +\frac{i}{4 \pi^{3}} \sum_{L^{\prime \prime}} \int d k_{0} \int k^{2} d k \frac{v_{L^{\prime} L^{\prime \prime}}\left(p_{0}^{\prime}, p^{\prime}, k_{0}, k ; s\right) t_{L^{\prime \prime} L}\left(k_{0}, k, p_{0}, p ; s\right)}{\left(\sqrt{s} / 2-E_{k}+i \epsilon\right)^{2}-k_{0}^{2}} .
\end{aligned}
$$

Here $t$ is the partial-wave decomposed T matrix and $v$ is the kernel of the $N N$ interaction, $E_{k}=$ $\sqrt{k^{2}+m^{2}}$. There is only one term in the sum for the singlet (uncoupled triplet) case $(L=J)$ and there are two terms for the coupled triplet case $(L=J \mp 1)$. We introduce the square of the total momentum $s=P^{2}=\left(p_{1}+p_{2}\right)^{2}$ and the relative momentum $p=\left(p_{1}-p_{2}\right) / 2\left[p^{\prime}=\left(p_{1}^{\prime}-p_{2}^{\prime}\right) / 2\right]$ (for details, see reference [2]).

Assuming the separable form (rank I) for the partial-wave decomposed kernels of NN interactions:

$$
v_{L^{\prime} L}\left(p_{0}^{\prime}, p^{\prime}, p_{0}, p ; s\right)=\lambda g^{\left[L^{\prime}\right]}\left(p_{0}^{\prime}, p^{\prime}\right) g^{[L]}\left(p_{0}, p\right),
$$

we can solve Eq. (1) and write for the $T$ matrix:

$$
t_{L^{\prime} L}\left(p_{0}^{\prime}, p^{\prime}, p_{0}, p ; s\right)=\tau(s) g^{\left[L^{\prime}\right]}\left(p_{0}^{\prime}, p^{\prime}\right) g^{[L]}\left(p_{0}, p\right),
$$

with the function $\tau(s)$ being given by

$$
\tau(s)=1 /\left(\lambda^{-1}+h(s)\right) .
$$

The function $h(s)$ has the following form:

$$
h(s)=\sum_{L} h_{L}(s)=-\frac{i}{4 \pi^{3}} \int d p_{0} \int p^{2} d p \sum_{L} \frac{\left[g^{[L]}\left(p_{0}, p\right)\right]^{2}}{\left(\sqrt{s} / 2-E_{p}+i \epsilon\right)^{2}-p_{0}^{2}} .
$$

The simplest separable function $g\left(p_{0}, p\right)$ which can be used is a covariant generalization of the non-relativistic Yamaguchi-type [5, 6] function:

$$
g\left(p_{0}, p\right)=\frac{1}{p_{0}^{2}-p^{2}-\beta^{2}+i \epsilon},
$$

where $\beta$ is a parameter.

\subsection{Modified Yamaguchi-type functions}

Let us consider the integral $h(s)$ (Eq. 5). Taking into account the pole structure of the propagators:

$$
p_{0}^{(1,2)}= \pm \sqrt{s} / 2 \mp E_{p} \pm i \epsilon
$$

and of the $g$ functions:

$$
p_{0}^{(3,4)}=\mp E_{\beta} \pm i \epsilon
$$

and using the Cauchy theorem, the $h(s)$ function can be written as follows:

$$
\frac{1}{2 \pi^{2}} \int p^{2} d p \frac{1}{\left(s / 4-\sqrt{s} E_{p}+m^{2}-\beta^{2}\right)^{2}} \frac{1}{\sqrt{s}-2 E_{p}+i \epsilon} .
$$

To calculate this integral one should analyze the factor $f=\left(s / 4-\sqrt{s} E_{p}+m^{2}-\beta^{2}\right)$ in the denominator as a function of $s$ : 
- if $2(m-\beta)<\sqrt{s}<2(m+\beta)$ then always $f<0$ and the function $1 / f^{n}$ is integrable for any integer $n$ and any $E_{p}$;

- for a bound state $\sqrt{s}=M_{d}=\left(2 m-\epsilon_{D}\right)$. Since for minimal $\beta_{\min }=0.2 \mathrm{GeV}$ always $\beta_{\min }>\epsilon_{D} / 2$ then the function $1 / f^{n}$ is integrable for any integer $n$ and any $E_{p}$;

- if $\sqrt{s}<2(m-\beta)$ or $\sqrt{s}>2(m+\beta)$ then $f$ can be positive and negative and $1 / f^{n}$ is non-integrable for even $n$ at any $E_{p}$.

The critical value $s^{c}=4(m+\beta)^{2}$ corresponds to the laboratory kinetic energy of $n p$-pair $T_{\text {lab }}^{c}=$ $4 \beta+2 \beta^{2} / m \simeq 4 \beta$. If $\beta_{\min }=0.2 \mathrm{GeV}$ then $T_{\text {lab }}^{\min }=0.8 \mathrm{GeV}$.

So, if we consider breakup processes of the deuteron such as photo-, electro- and nucleon-breakup Yamaguchi-functions can be used only if the laboratory kinetic energy of the NN-pair is less than $T_{\text {lab }}^{\mathrm{min}}$. To avoid this restriction we suggest to use Yamaguchi-type functions modified in the following way:

$$
g_{\mathrm{Y}}\left(p_{0}, p\right)=1 /\left(p_{0}^{2}-p^{2}-\beta^{2}\right) \longrightarrow g_{\mathrm{MY}}\left(p_{0}, p\right)=1 /\left(\left(p_{0}^{2}-p^{2}-\beta^{2}\right)^{2}+\alpha^{4}\right),
$$

here Y stands for the Yamaguchi and MY - for Modified Yamaguchi functions.

To work with the modified Yamaguchi-type functions the procedure of $p_{0}$ integration should be modified, too. This procedure is worthy of a special discussion. The poles of the $h(s)$ integral with the modified Yamaguchi-type functions are:

$$
\begin{aligned}
& p_{0}^{(3,4)}= \pm \sqrt{p^{2}+\beta^{2}+i \alpha^{2}}, \\
& p_{0}^{(5,6)}= \pm \sqrt{p^{2}+\beta^{2}-i \alpha^{2}} .
\end{aligned}
$$
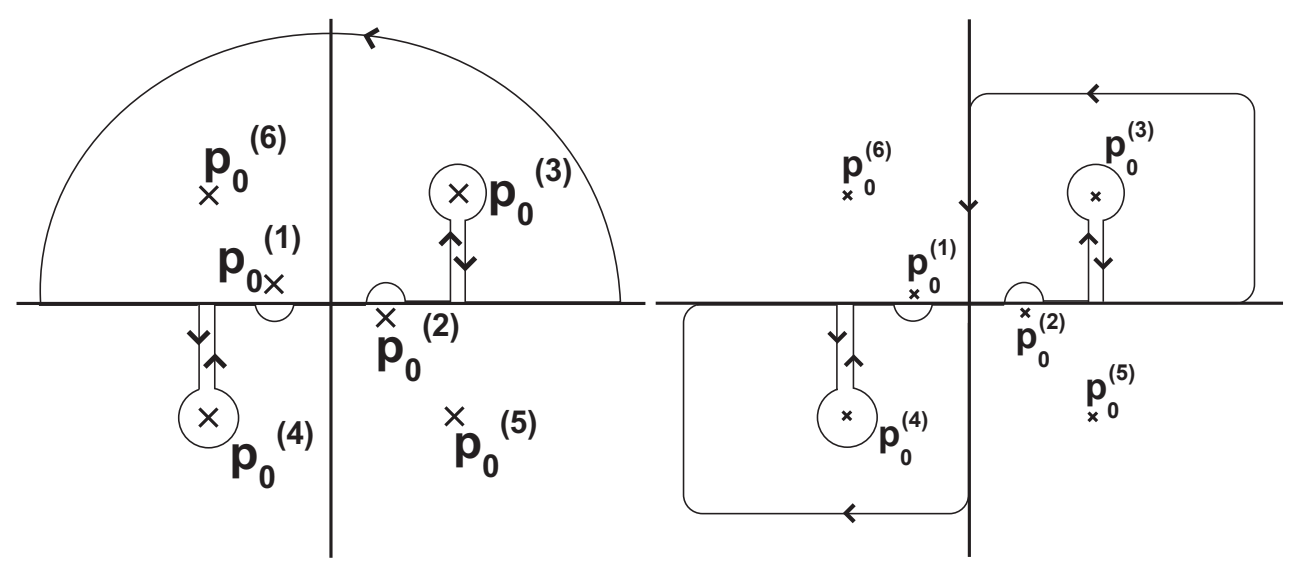

Figure 1. (a) Contour for the integration over $p_{0}$ according to the Cauchy theorem. (b) Contour for the integration over $p_{0}$ : the Wick rotation.

All poles and the contour of integration are pictured in Fig. 1(a,b). The idea how to choose the contour appeared owing to $[7,8]$. It is:

1. the contour must envelope the poles of $g$ form factors which will be inside the standard contour in the $\alpha \rightarrow 0$ limit. "Standard" means the contour used in the quantum field theory calculations 
with a propagator which has poles only on the real axis in the $p_{0}$ complex plane; one of them is circled from below and the other - from above. So, the path of integration is defined by an appropriate contour for the propagator;

2. the calculation over the presented path leads to a pure real contribution from the form factor poles and, therefore, to the unitary $S$ matrix (or the corresponding unitarity condition for the T matrix). We also obtain a correct transition to ordinary form factors of type $g \sim 1 /\left(p_{0}^{2}-p^{2}-\beta^{2}\right)^{2}$ in the $\alpha \rightarrow 0$ limit.

In general, the modified Yamaguchi-type functions can be written as:

$$
g_{i}^{[a]}\left(p_{0}, p\right)=\frac{\left(p_{c i}-p_{0}^{2}+p^{2}\right)^{n_{i}}\left(p_{0}^{2}-p^{2}\right)^{m_{i}}}{\left(\left(p_{0}^{2}-p^{2}-\beta_{1 i}^{2}\right)^{2}+\alpha_{1 i}^{4}\right)^{k_{i}}\left(\left(p_{0}^{2}-p^{2}-\beta_{1 i}^{2}\right)^{2}+\alpha_{1 i}^{4}\right)^{l_{i}}},
$$

where the parameters $-n_{i}, m_{i}, k_{i}, l_{i}$ (integer), $p_{c i}, \beta_{1 i}, \beta_{2 i}, \alpha_{1 i}, \alpha_{2 i}$ (real) depend on the channel $[a]$ under consideration. Such $g$ form factors are used to describe neutron-proton scattering observables (phase shifts, inelasticities, low-energy parameters and deuteron characteristics) for the total angular momentum $J=0,3$ in a wide energy range (see [9]-[13]).

\section{Acknowledgement}

One of the authors (S.G. Bondarenko) would like to thank the organizers of the International Conference "Mathematical Modeling and Computational Physics, 2017" (JINR, Dubna, July 3-7, 2017) for the invitation and opportunity to present our results.

This work was partially supported by the Russian Foundation for Basic Research grant $N$ o $16-02$ 00898 .

\section{References}

[1] E.E. Salpeter and H.A. Bethe, Phys. Rev. 84, 1232 (1951)

[2] S.G. Bondarenko, V.V. Burov, A.V. Molochkov, G.I. Smirnov, and H. Toki, Prog. Part. Nucl. Phys. 48, 449 (2002)

[3] L. Mathelitsch, W. Plessas, and W. Schweiger, Phys. Rev. C26, 65 (1982)

[4] J. Haidenbauer and W. Plessas, Phys. Rev. C30, 1822 (1984)

[5] Y. Yamaguchi, Phys. Rev. 95, 1628 (1954)

[6] Y. Yamaguchi, Y. Yamaguchi, Phys. Rev. 95, 1635 (1954)

[7] R.E. Cutkosky, P.V. Landshoff, D.I. Olive, and J.C. Polkinghorne, Nucl. Phys. B12, 281 (1969)

[8] T.D. Lee and G.C. Wick, Nucl. Phys. B9, 209 (1969)

[9] S.G. Bondarenko, V.V. Burov, W.-Y. Pauchy Hwang, and E.P. Rogochaya, Nucl. Phys. A832, $233(2010)$

[10] S.G. Bondarenko, V.V. Burov, W.-Y. Pauchy Hwang, and E.P. Rogochaya, Nucl. Phys. A848, 75 (2010)

[11] S.G. Bondarenko, V.V. Burov, and E.P. Rogochaya, Phys. Lett. B705, 264-268 (2011); Nucl. Phys. Proc. Suppl. 219-220, 126-129 (2011)

[12] S.G. Bondarenko, V.V. Burov, and E.P. Rogochaya, Nucl. Phys. Proc. Suppl. 245, 291-297 (2013)

[13] S.G. Bondarenko, V.V. Burov, S.E. Kemelzhanova, E.P. Rogochaya, and N. Sagimbaeva, Proceedings of the 12th International Workshop "Relativistic Nuclear Physics from Hundreds of MeV to TeV" (Slovak Republic, Stara Lesna, June 16-20, 2014) 